\title{
Integrated Analysis of the miRNA-mRNA Regulatory Network Involved in the HIV-Associated Neurocognitive Disorder
}

\author{
Chang Liu ${ }^{1, *}$, Qian Ding ${ }^{1}$ and Xiaohong Kong ${ }^{1, *}$ \\ 1 School of Medicine, Nankai University, No. 94, Weijin Road, Nankai District, Tianjin, China; 300071; \\ (2120181369@mail.nankai.edu.cn) Q.D. \\ * Correspondence: CL, changliu@nankai.edu.cn; XK, kongxh@nankai.edu.cn
}

\begin{abstract}
HIV-associated neurocognitive disorder (HAND) is an array of neurocognitive changes associated with HIV infection, and the roles of microRNAs in HAND are not completely revealed yet. Based on published data and publicly available databases, we constructed an integrated miRNA-mRNA network involved in HAND. Bioinformatics analyses, including gene ontology, network analysis, and KEGG pathway analysis, were applied for further study of the network and the genes of the network. The axon guidance KEGG pathway, three genes NTNG1, EFNB2, CXCL12, and 17 miRNAs which regulates them, are spotlighted in our study. This study provides new perspectives to the knowledge of miRNAs' roles in the process of HAND, and our findings provided potential therapeutic targets and clues of HAND.
\end{abstract}

Keywords: miRNA; mRNA; HIV; network; bioinformatics; HAND; viral infection; CNS damage

\section{Introduction}

Although the HIV/AIDS epidemic remains a significant global health challenge[1], antiretroviral therapy (ART) has transformed HIV infection from a fatal disease into a chronic illness[2]. ART achieves HIV suppression, but HIV-associated neurocognitive disorder (HAND) has been one of the most prevalent comorbidities in the era of ART [3]. HAND comprises a spectrum of conditions ranging from asymptomatic neurocognitive impairment to mild neurocognitive disorder to HIV-associated dementia (HAD), the clinical correlate of HIV encephalitis [4]. During HIV infection, neuronal damage is likely a bystander effect of infected cells since neurons are not HIV-permissive cells [5]. HIVinfected macrophages and lymphocytes can cross the blood-brain-barrier and infect glial cells [6], so HIV infection is restricted to microglia and astrocytes in the central nervous system (CNS) where resident lymphoid cells are absent [7]. In the CNS, HIV-infected cells release a plethora of host and viral molecules including chemokines, cytokines and viral proteins; these molecules promote neuroinflammation even during ART while HIV replication is undetectable [8]. Furthermore, some studies have reported that shed or secreted HIV proteins, such as gp120, Tat and Vpr, participate in the neuropathogenesis of HIV [911].

Because neurons are not productively infected by HIV, neuronal damage is mainly through indirect mechanisms [12]. CNS neurons are vulnerable to damage by HIV viral proteins, and the vulnerability is largely mediated through the presence of several neuronal cellular receptors, such as chemokine receptors (CCR5 or CXCR4), the low-density lipoprotein receptor related protein (LRP), the N-methyl-D-Aspartate receptors (NMDAR), and the dopamine transporter [13-16]. HIV envelope protein gp120 incites activation of chemokine receptors on neurons and triggers elevation of intracellular $\mathrm{Ca}^{2+}$ leading to apoptosis [17]. HIV transcriptional activator protein Tat can also bind LRP in neurons, causing LRP internalization and inducing apoptosis $[18,19]$. Furthermore, because ART does not impact levels of HIV Tat and the CNS is not accessible to the ART regiments, Tat has been regarded as a mediator of HAND [20]. However, the detailed 
molecular mechanism how HIV damages neurons need further exploration. Recently, accumulating evidence has implicated that microRNAs (miRNAs) also play import roles in this process[21-23].

MiRNAs are a class of endogenous, small, non-coding RNAs of 18-26 bp; and miRNAs can regulate post-transcriptional protein expression via interaction with 3'UTR region of the target genes' message RNAs (mRNAs)[24,25]. The CNS is the most complex organ of mammals, and houses a remarkable diversity of miRNAs[26], therefore the CNS is an interesting study target for miRNA studies[27]. The CNS miRNAs play an important role in regulating gene expression during neuronal development, from neurogenesis to synaptogenesis, as well as in maintaining normal neuron function[28-30]. Moreover, several reports have suggested that miRNAs are involved in HIV-related neural injury: Rahimian et al. reported that HIV Tat significantly induces the expression of miR-132, downregulates target genes of miR-132 in astrocytes and neurons, and causes neurite shortening[31]; Bagashev et al. found that Tat deregulates the p73 pathway and causes neuronal dysfunction through miR-196a[32]; miR-142 is increased in neurons and myeloid cells in the brain of HAND patients, and indirectly downregulates MAOA to change dopaminergic neurotransmission[33]; miR-21 is significantly upregulated in the brain of simian immunodeficiency virus encephalitis (SIVE) monkey model, and it specifically targets MEF2C to induce neuronal dysfunction and neurodegeneration[34]. The abovementioned studies indicate that HIV can manipulate the expression of neuronal genes, via modulating the expression of particular microRNAs, and miRNAs may serve as key elements in gene regulatory networks in HAND.

In our study, an integrated miRNA-mRNA regulatory network was constructed and studied. We collated the miRNAs whose expressions have been reported changing significantly in HAND[22,23,31-40]. These miRNAs were divided into two different groups: the upregulated group and the downregulated group. The potential miRNA-target genes were predicted by three miRNA-target prediction public databases[41-43]. The union of each miRNA's potential miRNA-target genes in different database was constructed, so a mini miRNA-target genes of HAND database was established. In order to construct a miRNA-mRNA regulatory network involved in HAND, a Gene Expression Omnibus (GEO, https://www.ncbi.nlm.nih.gov/geo/) dataset was reanalyzed to obtain the differential expression genes (DEGs) in neurons effected by HIV. These DEGs were used as a screening condition to construct the miRNA-mRNA network; then the network was analyzed and demonstrated by Cytoscape[44] and $\mathrm{R}$ packages[45]. These miRNA-target genes in the network were performed to construct a protein-protein-interaction (PPI) network, and the genes of the PPI network were applied KEGG pathway enrichment. The axon guidance pathway (hsa04360) was enriched, the miRNAs and mRNAs/genes of our network participating this pathway were found. Our study generated a holistic picture of miRNA-mRNA regulatory involved in HAND, and some potential important miRNAs and mRNAs/genes of the network could be regarded as therapeutic targets or agents for the treatment of HAND in the future.

\section{Results}

\subsection{Identification and Functional Analysis of DEGs Associated with HAND}

In order to identify the significant differentially expressed genes (DEGs) associated with HAND, GSM1045805, GSM1045806, GSM1045807, GSM1045808 in GSE44265 were reanalyzed by $\mathrm{R}$ software. As shown in figure $1 \mathrm{~A}$, a volcano plot showed the genes' expression changing in differentiated SH-5Y cells after treatment with supernatant from HIV infected U-937; the red dots indicated the significantly changing genes with more than a 2-fold change and an adjusted $p$ value of less than 0.05 ; and the drastically changing 
DEGs with more than 5.657-fold change (or $2.5 \log 2$ fold changing) were annotated by gene name. We called these drastically changing mRNAs/genes representative DEGs.

A

C

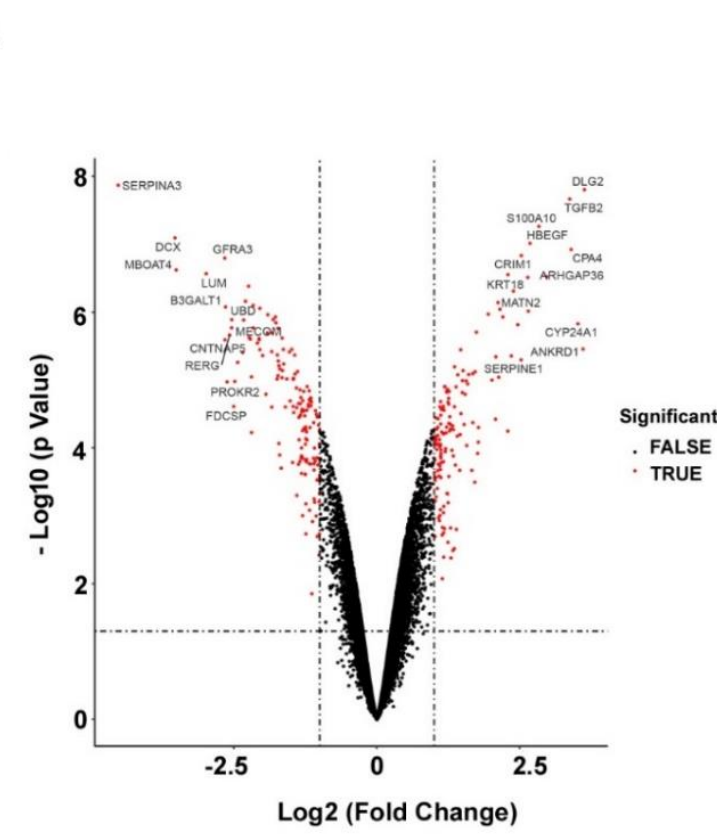

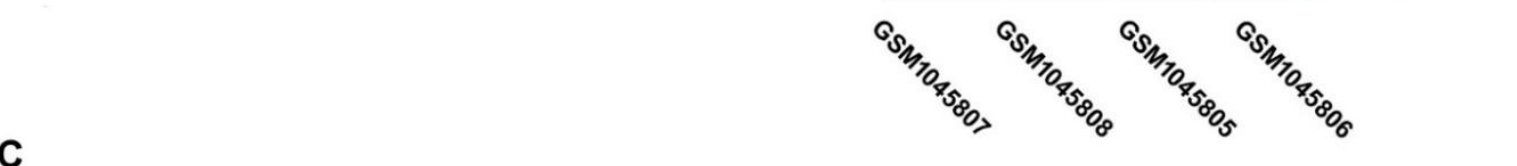

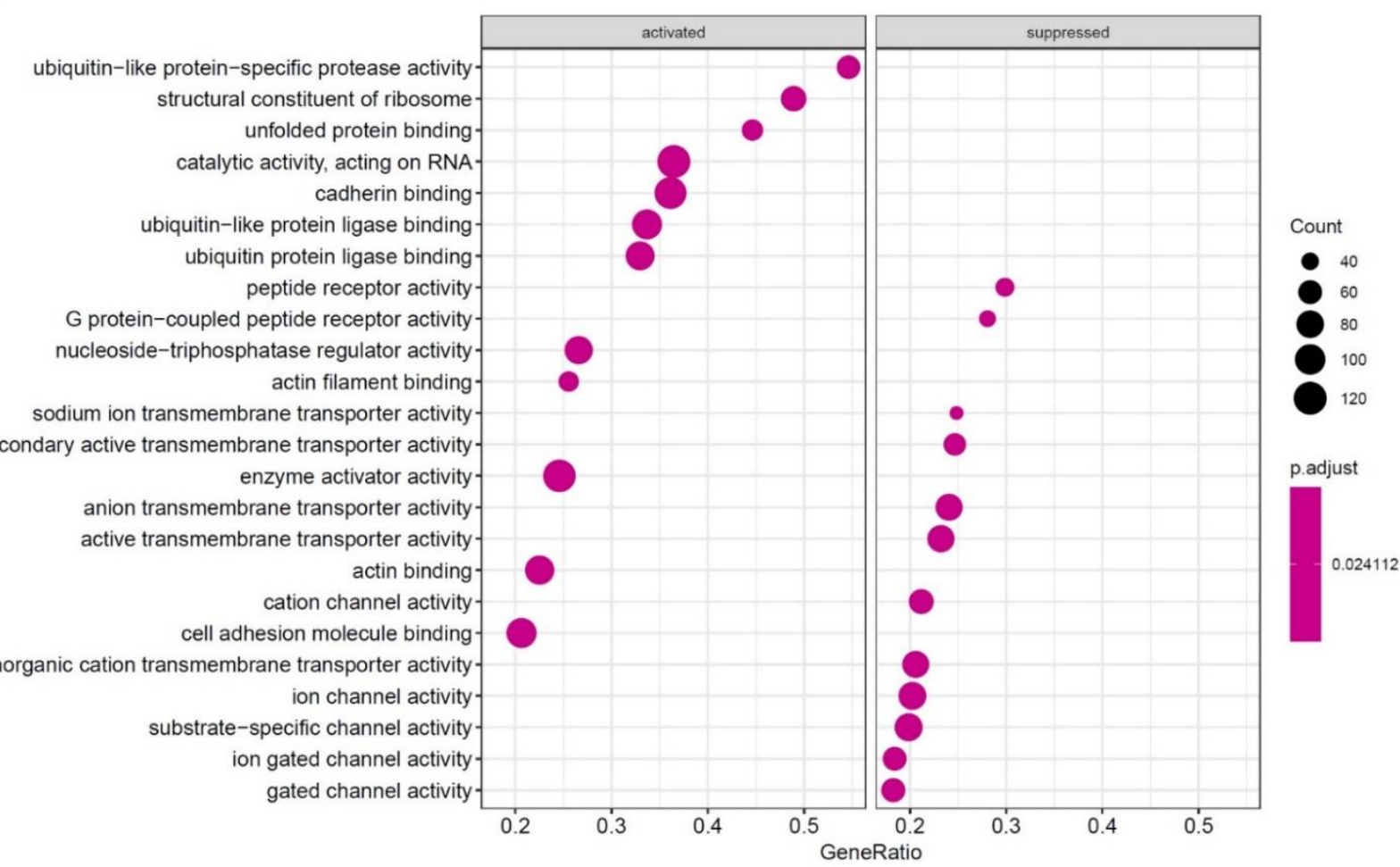

Figure 1. Analysis of DEGs between HIV supernatant treating and mock-treating differentiated SH-5Y cells. (A) Volcano plot of all DEGs. (B) Heatmap of representative DEGs. (C) Molecular function Go analysis of all 296 significant DEGs which were separated into the activated group and the suppressed group. 
For more detailed information as shown in figure $1 \mathrm{~B}$, there are 12 representative upregulated expression mRNAs/genes and 12 representative downregulated expression mRNAs/genes. The cluster heatmap of these representative changing DEGs is presented in figure 1 B. To further understand the molecular functions of DEGs, all 296 significant DEGs were separated into two group: the upregulated/activated mRNA-gene group (142 genes, the activated group) and the downregulated/suppressed mRNA-gene group (154 genes, the suppressed group). These genes of the two groups were respectively taken GO molecular function (GO-MF) enrichment analysis. As showcased in figure $1 \mathrm{C}$, some cellular channel activities, such as cation channel, ion channel, substrate-specific channel, ion gated channel and gated channel, are significantly enriched in the suppressed group. The suppression of these channels' activity obviously affects the functions of neurons in CNS.

\subsection{Construction of the miRNA-mRNA Net Involved in HAND}

According to the published papers about miRNA and HIV associated neural damage, 101 upregulated miRNA and 91 downregulated miRNAs were selected and their miRNA target mRNAs/genes were searched and predicted in three public web databases (miRtarbase database[41], miRDB database[42] and Targetscan database[43]). The upregulatedmiRNA-downregulated-mRNA pairs were filtered with previously constructed downregulated/suppressed mRNAs/gene group (154 genes); and downregulated-miRNAmRNA pairs were filtered with the upregulated/activated mRNA/gene group (142genes) correspondingly. Finally, an upregulated-miRNA-downregulated-mRNA network, which contains 51 miRNAs and 136 mRNAs/genes and 1458 regulation connections, was constructed; meanwhile, a downregulated-miRNA-upregulated-mRNA network, which contains 88 miRNAs and 129 mRNAs/genes and 1981 regulation connections, was also constructed. These two complete miRNA-mRNA regulation pair tables were provided as supplementary materials.

A

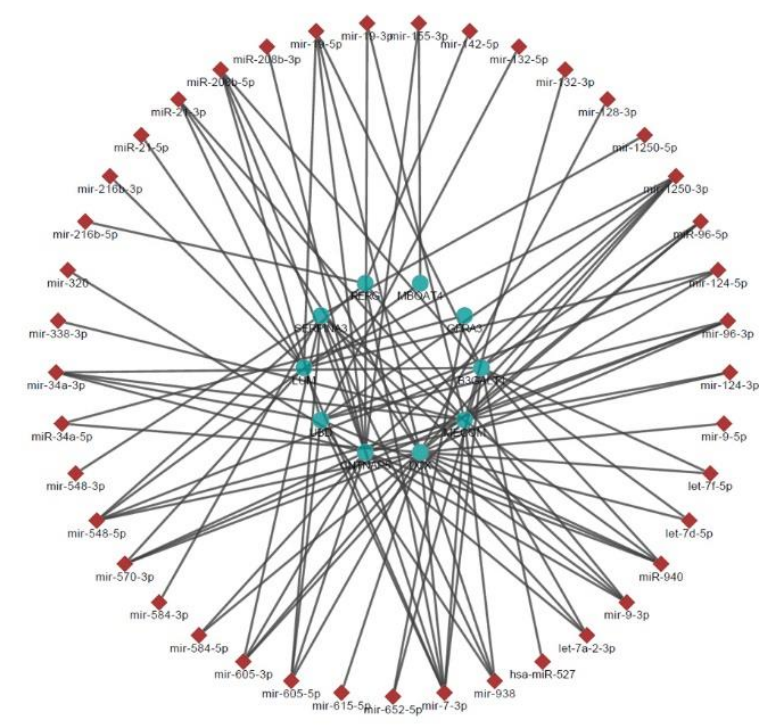

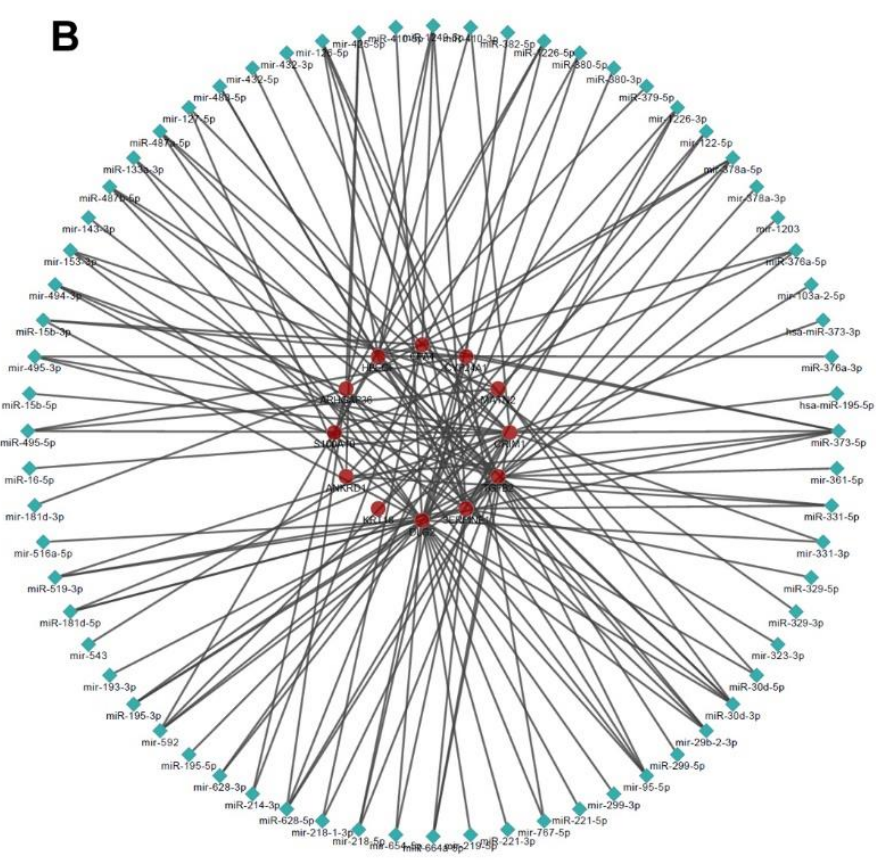

Figure 2. The representative miRNA-mRNA pairs networks. (A) The representative upregulated-miRNA-downregulatedmRNA network. (B) The representative downregulated-miRNA-upregulated-mRNA network.

To show the features of the miRNA-mRNA networks intuitively, we constructed two mini networks filtered with representative DEGs as mentioned above. As shown in figure 2, upregulated miRNAs were indicated as red diamonds; downregulated mRNAs/genes were indicated as green dots; downregulated miRNAs were indicated as green diamonds; 
upregulated mRNAs/genes were indicated as red dots; pairs were connected by strain lines. The connectional characteristics of a certain miRNA or mRNA/gene in the network were significantly different. Some miRNAs, such as has-miR-527 or mir-615-5p only regulated one certain target mRNA/gene in the representative network, while some miRNAs, such as mir-7-3p or miR-1250-3p, regulated more than 5 target mRNAs/genes; the target mRNAs/genes behaved similarly. These miRNAs or mRNAs/genes, which had more connections with other genes or miRNAs, may play a more important role in the network comparing with other miRNAs or genes. We called them key miRNAs or mRNA/genes in the miRNA-mRNA network. In the network analysis, the degree of a node, which is the number of its neighbors, shows this characteristic; a node with high degree has more edges/connection than a low degree node. The node degree analysis was carried on the whole miRNA-gene network, finally top 10 upregulated miRNAs, downregulated mRNAs/genes, downregulated miRNAs and upregulated mRNAs/genes were listed in table 1.

Table 1. Top 10 up/down miRNA/mRNA in the miRNA-mRNA network base on degree analysis

\begin{tabular}{cccc}
\hline $\begin{array}{c}\text { Upregulated miR- } \\
\text { NAs }\end{array}$ & $\begin{array}{c}\text { Downregulated } \\
\text { miRNAs }\end{array}$ & $\begin{array}{c}\text { Upregulated } \\
\text { mRNAs/genes }\end{array}$ & $\begin{array}{c}\text { Downregulated } \\
\text { mRNAs/genes }\end{array}$ \\
\hline mir-570-3p & mir-95-5p & LPP & CNNM2 \\
mir-7-3p & miR-373-5p & STX7 & FUT9 \\
mir-1250-3p & mir-126-5p & DCLK1 & SLC8A1 \\
miR-940 & miR-30d-3p & CCDC80 & CLSTN2 \\
mir-548-5p & miR-195-3p & ERBB4 & IGSF3 \\
miR-208b-5p & miR-329-5p & CADM2 & NEBL \\
mir-9-3p & mir-29b-2-5p & PARVA & RAB27B \\
mir-19-5p & miR-519-3p & NRXN3 & CACNA1C \\
mir-548-3p & mir-103a-2-5p & PLA2G16 & DCX \\
mir-124-5p & miR-380-3p & DNAJB4 & BAMBI \\
\hline
\end{tabular}

\subsection{Functional Exploration of the miRNA-mRNA Net Involved in HAND}

In order to explore the potential biological function of the genes in the miRNAmRNA net, 136 downregulated mRNAs/genes and 129 upregulated mRNAs/genes were analyzed by ClueGo plugin of Cytoscape. As shown in figure $3 \mathrm{~A}$, a protein-protein interaction (PPI) network including 34 downregulated mRNAs/genes, 18 upregulated mRNA/genes and 8 enriched genes, was constructed. The relations between these genes were activation or inhibition which was indicated as green arrows or red arrows respectively. Based on the results of the PPI net, all 60 mRNAs/genes (34 downregulated, 18 upregulated, and 8 enriched) were used to find the key KEGG pathways. Several important signaling pathways were significantly enriched, including PI3K-Akt signaling pathway (hsa04151), Wnt signaling pathway (hsa04310), MAPK signaling pathway (hsa04010), TGF-beta signaling pathway (hsa04350), NF-kappa B signaling pathway (hsa04064). These results implicated that miRNAs participates in multiple cellular signaling pathways. 


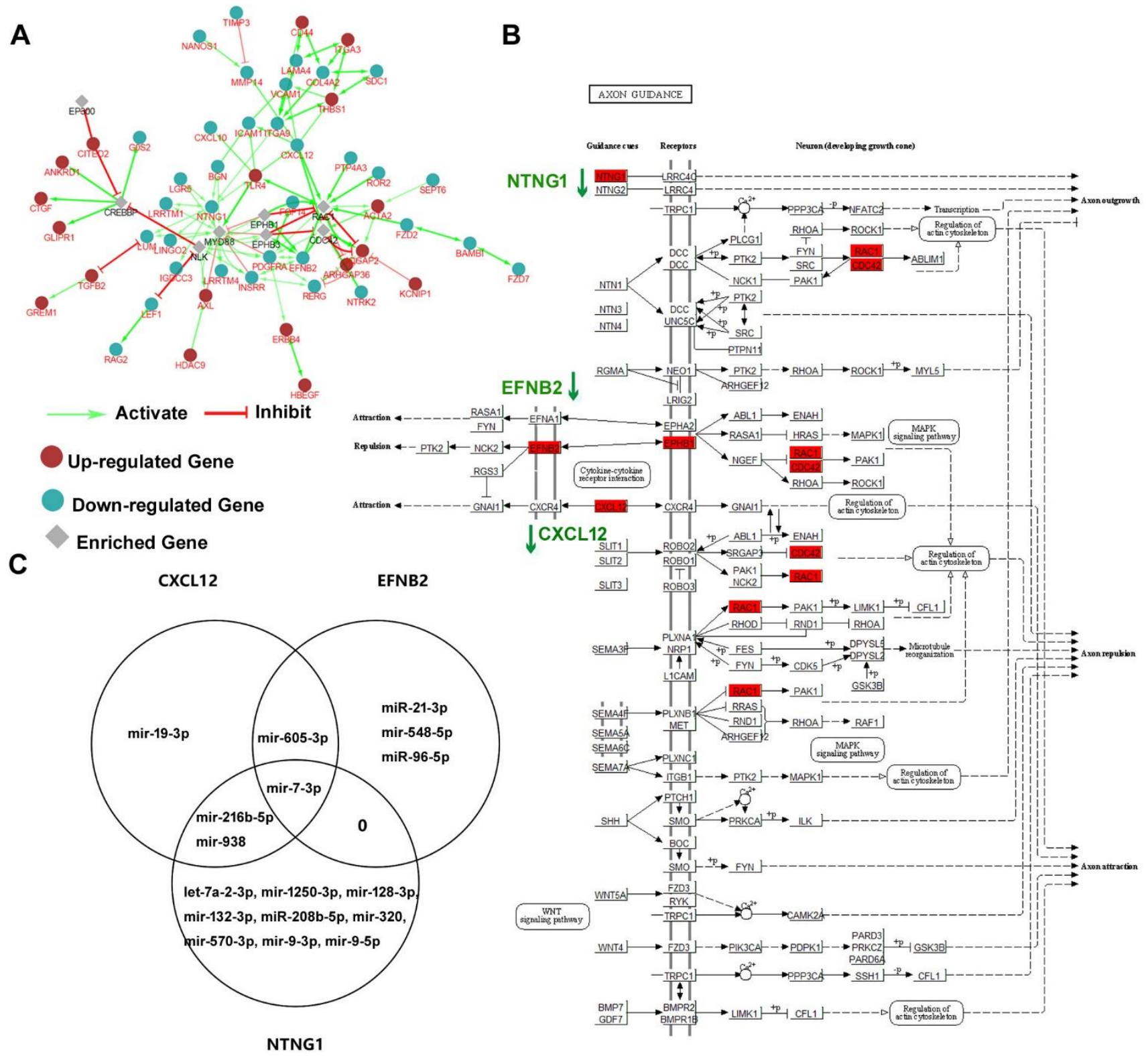

Figure 3. Functional exploration of the miRNA-mRNA network. (A) The PPI network of the mRNAs/genes in the miRNAmRNA network. (B) Visualization of the axon guidance (hsa04360) KEGG pathway. (C) Venn diagram showing miRNAs which regulate the 3 genes participating in the axon guidance pathway.

Furthermore, the axon guidance pathway (hsa04360) was also significantly enriched; there were 7 genes ( $3 \mathrm{mRNAs} /$ genes in the miRNA-mRNA network and 4 enriched genes in the PPI network) participating in this pathway. All seven genes were marked red in figure $3 \mathrm{~B}$. Three mRNAs/genes in the miRNA-mRNA network were NTNG1, EFNB2, and CXCL12, which were all downregulated in the miRNA-mRNA pairs network. For clarity, all these 3 genes were written in green and down arrows indicating downregulation. We also queried the miRNAs for these 3 genes with our miRNA-mRNA network, those miRNAs which regulate these 3 genes were shown in a Venn diagram of figure $3 \mathrm{C}$. There were 5 miRNAs regulating EFNB2, 5 miRNAs regulating CXCL12, and 12 miRNAs regulating NTNG1. A single miRNA may regulate multiple mRNAs/genes; for example, mir7-3p can regulate NTNG1, EFNB2, and CXCL12, as indicated in figure 3 C. 


\section{Discussion}

In our study, we constructed and analyzed an integrated miRNA-mRNA regulatory network involved the HAND. The miRNA information is acquired from 12 published papers about the expression changing of miRNAs during HIV-associated neuron damage. Among these studies, some studies were focused on some specific miRNAs, and some studies were general miRNA screening; some were clinical case studies, and some were mechanism studies at the cellular level or animal models. We collected these miRNAs expecting to explore undiscovered molecular mechanisms. The mRNAs/genes information came from reanalyzing or reusing the GSE44265 GEO dataset. Based on our experimental design requirements, finally four samples (GSM1045805, GSM1045806, GSM1045807, GSM1045808) were chosen. GSM1045807 and GSM1045808 are differentiated SH-5Y cells treated with supernatant from HIV infected U-937; GSM1045805 and GSM1045806 are normal differential SH-5Y cells as the negative control group. Differentiated SH-5Y cells are neuronal cell line cells which are widely used instead of primary neurons. Considering the difficulty of getting the clinical tissue samples of HAND patients (maybe impossible to obtain samples from early patients), we used DEGs between these cells reflecting the mRNAs/genes changing in neurons of HAND patients.

After the miRNA-mRNA network involved HAND was constructed, we gave the key miRNAs and mRNAs depending the number of their neighbors/connections. This choice is somewhat arbitrary. Hausser and Zavolan has reviewed that a miRNA binds to hundreds of sites across the transcriptome, so target mRNAs/genes are relatively excess comparing with miRNAs[46]. If we want to focus on the genes which are regulated by miRNAs, these mRNAs/genes regulated by many miRNAs simultaneously should be priorly paid close attention. On another hand, these miRNAs, which regulates more mRNAs/genes, have a greater possibility to affect a pathway to exercise their biological functions. For example, mir-7-3p, which was in our table 2, was also found to regulated 3 target mRNAs/genes in the enriched KEGG pathway in figure 3.

Finally, based on our constructed miRNA-mRNA network, we found a KEGG pathway__axon guidance pathway (hsa04360) through PPI network exploration and KEGG pathway enrichment. Some studies support our findings. A parallel genome-wide mRNA and miRNA profiling of the frontal cortex of HIV patients with and without HIV-associated dementia has shown the role of axon guidance and downstream pathways in HIVmediated neurodegeneration, and miR-137, 153 and 218 targeted neurodegeneration related pathways[22]. Our study focused on the same axon guidance pathway, but different miRNAs and targets are provided. CXCL12, which was one of three genes in the pathway, and its receptor CXCR4, which is also the coreceptor of HIV, participate in the pathogenesis of CNS disorders such as HIV-associated encephalopathy, brain tumor, stroke and multiple sclerosis[47]. Furthermore, one study of exosomal miRNAs associated with neuropsychological performance in individuals with HIV infection on antiretroviral therapy has shown that a high proportion of differentiating exosomal miRNAs target the axon guidance KEGG pathway[48].

In summary, based on published data and publicly available databases, we constructed an integrated miRNA-mRNA network involved in HAND. On the basis of the miRNA-mRNA network, some key miRNAs and mRNAs/genes (listed in table 1) are highlighted to some investigators who are interested in HIV-related neuronal damage. Furthermore, the axon guidance pathway, three genes of our network involved in this pathway, and those miRNAs which regulate them in our miRNA-mRNA network, should be also concerned by some relevant researchers. All of our findings provide new perspectives to the understanding of miRNAs and mRNAs underlying the HAND process, and experimental verification is needed to validate our predictions. 


\section{Materials and Methods}

\subsection{GEO Data Processing}

The dataset GSE44265 provided by Sawaya BE. et al. (https://www.ncbi.nlm.nih.gov/geo/query/acc.cgi?acc=GSE44265) [39] was downloaded from the GEO database. In the dataset, GSM1045807 and GSM1045808 are differentiated SH-5Y cells treated with supernatant from HIV infected U-937; GSM1045805 and GSM1045806 are normal differential SH-5Y cells as the negative control group. All samples were hybridized on the Affymetrix Human Gene 1.1 ST Array (Affymetrix, Santa Clara, CA, USA). All of the raw data were processed using affy and related R packages with Robust Multi-array Average approach for background normalization as per the package instruction[49]. Differentially expressed genes (DEGs) were selected according to changing folds more than 2 and $p$ value less than 0.05 . The DEGs were divided into the upregulated DEG group and the downregulated DEG group.

\subsection{MiRNA Selection and miRNA-Target Gene Prediction}

101 upregulated miRNAs and 91 downregulated miRNAs were selected according to 12 published papers about HIV neural damage. To predict the miRNA-target genes of the corresponding miRNA, miRtarbase database (http://mirtarbase.mbc.nctu.edu.tw)[41], miRDB database (http://mirdb.org/)[42] and Targetscan database (www.targetscan.org)[43] three public databases were applied to search the target genes. The union of all target genes from three databases was selected as the candidate dataset of miRNAtarget genes of the corresponding miRNA.

\subsection{Construction of miRNA-mRNA Regulatory Network}

In order to construct the miRNA-mRNA regulatory network, upregulated miRNAs and their miRNA-target genes were intersected with genes of the downregulated DEG group; downregulated miRNAs and their miRNA-target genes were intersected with genes of the upregulated DEG group. Finally, 1458 upregulated-miRNA-and-downregulated-mRNA pairs and 1981 downregulated-miRNA-and-upregulated-mRNA pairs were selected to construct the miRNA-mRNA regulatory network involved HAND by Cytoscape software (version 3.7.1)[44].

\subsection{Identification of Key miRNAs and $m R N A s / G e n e s$ in miRNA-mRNA Regulatory Network}

The important nodes (key miRNAs and genes) of the miRNA-mRNA regulatory network were predicted and explored by Cytoscape "cytoHubba" plugin[50]. In upregulated-miRNA-downregulated-mRNA network, top 10 ranked net degrees upregulated miRNAs and top 10 ranked net degrees downregulated mRNAs/genes were identified as key upregulated miRNAs and key downregulated mRNAs. Similarly, 10 key downregulated miRNAs and 10 key upregulated mRNAs were identified in the downregulatedmiRNA-upregulated-mRNA network.

\subsection{PPI Network Construction}

To predict the biofunctions and interactions of the genes in the miRNA-mRNA network, the Cytoscape "ClueGo" plugin was applied[51,52]. The maximum sub PPI network which contained the largest number of the net genes was selected.

\subsection{KEGG analysis and the pathway visualization}

KEGG analysis was performed using the KEGG pathway database (https:/www.genome.jp/kegg/pathway.html)[53]. To show the key KEGG pathway, an R/Bioconductor package named Pathview was applied to visualize the concerned KEGG pathway[45].

\subsection{Venn diagram}

In order to show the miRNAs against 3 genes in the key KEGG pathway, an online venn diagram tool Venny2.1 (https://bioinfogp.cnb.csic.es/tools/venny/) was apply.

Supplementary Materials: The following supporting information can be downloaded at: www.mdpi.com/xxx/s1, Table S1: Down-miRNA-up-mRNA pairs; Table S2: Up-miRNA-downmRNA pairs. 


\begin{abstract}
Author Contributions: Conceptualization, L.C. and K.X.; methodology, L.C and D.Q.; software, L.C.; investigation, L.C.; data curation, D.Q.; writing-original draft preparation, L.C.; writingreview and editing, K.X.; visualization, L.C.; supervision, K.X.; project administration, K.X.; funding acquisition, L.C. All authors have read and agreed to the published version of the manuscript.
\end{abstract}

Funding: This research was funded by Natural Science Foundation of Tianjin Municipal Science and Technology Commission, grant number 18JCYBJC28600 and the National Natural Science Foundation of China, grant number 81601996.

Institutional Review Board Statement: Not applicable.

Informed Consent Statement: Not applicable.

Data Availability Statement: Not applicable.

Acknowledgments: The authors are especially grateful to Biotrainee (http://www.biotrainee.com/) for much useful help in bioinformation analysis.

Conflicts of Interest: The authors declare no conflict of interest.

\title{
References
}

1. Moranguinho, I.; Valente, S.T. Block-And-Lock: New Horizons for a Cure for HIV-1. Viruses 2020, 12, doi:10.3390/v12121443.

2. Battivelli, E.; Dahabieh, M.S.; Abdel-Mohsen, M.; Svensson, J.P.; Tojal Da Silva, I.; Cohn, L.B.; Gramatica, A.; Deeks, S.; Greene, W.C.; Pillai, S.K.; et al. Distinct chromatin functional states correlate with HIV latency reactivation in infected primary CD4(+) T cells. eLife 2018, 7, doi:10.7554/eLife.34655.

3. Wei, J.; Hou, J.; Su, B.; Jiang, T.; Guo, C.; Wang, W.; Zhang, Y.; Chang, B.; Wu, H.; Zhang, T. The Prevalence of FrascatiCriteria-Based HIV-Associated Neurocognitive Disorder (HAND) in HIV-Infected Adults: A Systematic Review and MetaAnalysis. Front Neurol 2020, 11, 581346, doi:10.3389/fneur.2020.581346.

4. Achim, C.L.; Wang, R.; Miners, D.K.; Wiley, C.A. Brain viral burden in HIV infection. J Neuropathol Exp Neurol 1994, 53, 284294, doi:10.1097/00005072-199405000-00010.

5. $\quad \mathrm{Ru}$, W.; Liu, X.; Bae, C.; Shi, Y.; Walikonis, R.; Mo Chung, J.; Tang, S.J. Microglia Mediate HIV-1 gp120-Induced Synaptic Degeneration in Spinal Pain Neural Circuits. The Journal of neuroscience : the official journal of the Society for Neuroscience 2019, 39, 8408-8421, doi:10.1523/JNEUROSCI.2851-18.2019.

6. Sachan, V.; Lodge, R.; Mihara, K.; Hamelin, J.; Power, C.; Gelman, B.B.; Hollenberg, M.D.; Cohen, E.A.; Seidah, N.G. HIVinduced neuroinflammation: impact of PAR1 and PAR2 processing by Furin. Cell Death Differ 2019, 26, 1942-1954, doi:10.1038/s41418-018-0264-7.

7. Churchill, M.; Nath, A. Where does HIV hide? A focus on the central nervous system. Curr Opin HIV AIDS 2013, 8, 165-169, doi:10.1097/COH.0b013e32835fc601.

8. Clifford, D.B. HIV-associated neurocognitive disorder. Curr Opin Infect Dis 2017, 30, 117-122, doi:10.1097/QCO.0000000000000328.

9. Omeragic, A.; Kara-Yacoubian, N.; Kelschenbach, J.; Sahin, C.; Cummins, C.L.; Volsky, D.J.; Bendayan, R. Peroxisome Proliferator-Activated Receptor-gamma agonists exhibit anti-inflammatory and antiviral effects in an EcoHIV mouse model. Sci Rep 2019, 9, 9428, doi:10.1038/s41598-019-45878-6.

10. Carvallo, L.; Lopez, L.; Fajardo, J.E.; Jaureguiberry-Bravo, M.; Fiser, A.; Berman, J.W. HIV-Tat regulates macrophage gene expression in the context of neuroAIDS. PloS one 2017, 12, e0179882, doi:10.1371/journal.pone.0179882.

11. Guha, D.; Nagilla, P.; Redinger, C.; Srinivasan, A.; Schatten, G.P.; Ayyavoo, V. Neuronal apoptosis by HIV-1 Vpr: contribution of proinflammatory molecular networks from infected target cells. Journal of neuroinflammation 2012, 9, 138, doi:10.1186/1742-2094-9-138.

12. Kim, S.H.; Smith, A.J.; Tan, J.; Shytle, R.D.; Giunta, B. MSM ameliorates HIV-1 Tat induced neuronal oxidative stress via rebalance of the glutathione cycle. Am J Transl Res 2015, 7, 328-338. 
13. Cull-Candy, S.; Brickley, S.; Farrant, M. NMDA receptor subunits: diversity, development and disease. Current opinion in neurobiology 2001, 11, 327-335.

14. Hayashi, H.; Campenot, R.B.; Vance, D.E.; Vance, J.E. Apolipoprotein E-containing lipoproteins protect neurons from apoptosis via a signaling pathway involving low-density lipoprotein receptor-related protein-1. The Journal of neuroscience : the official journal of the Society for Neuroscience 2007, 27, 1933-1941, doi:10.1523/JNEUROSCI.5471-06.2007.

15. Chang, L.; Wang, G.J.; Volkow, N.D.; Ernst, T.; Telang, F.; Logan, J.; Fowler, J.S. Decreased brain dopamine transporters are related to cognitive deficits in HIV patients with or without cocaine abuse. NeuroImage 2008, 42, 869-878, doi:10.1016/j.neuroimage.2008.05.011.

16. Rao, V.R.; Ruiz, A.P.; Prasad, V.R. Viral and cellular factors underlying neuropathogenesis in HIV associated neurocognitive disorders (HAND). AIDS research and therapy 2014, 11, 13, doi:10.1186/1742-6405-11-13.

17. Catani, M.V.; Corasaniti, M.T.; Navarra, M.; Nistico, G.; Finazzi-Agro, A.; Melino, G. gp120 induces cell death in human neuroblastoma cells through the CXCR4 and CCR5 chemokine receptors. Journal of neurochemistry 2000, 74, $2373-2379$.

18. Liu, Y.; Jones, M.; Hingtgen, C.M.; Bu, G.; Laribee, N.; Tanzi, R.E.; Moir, R.D.; Nath, A.; He, J.J. Uptake of HIV-1 tat protein mediated by low-density lipoprotein receptor-related protein disrupts the neuronal metabolic balance of the receptor ligands. Nature medicine 2000, 6, 1380-1387, doi:10.1038/82199.

19. Eugenin, E.A.; King, J.E.; Nath, A.; Calderon, T.M.; Zukin, R.S.; Bennett, M.V.; Berman, J.W. HIV-tat induces formation of an LRP-PSD-95- NMDAR-nNOS complex that promotes apoptosis in neurons and astrocytes. Proceedings of the National Academy of Sciences of the United States of America 2007, 104, 3438-3443, doi:10.1073/pnas.0611699104.

20. Periyasamy, P.; Thangaraj, A.; Guo, M.L.; Hu, G.; Callen, S.; Buch, S. Epigenetic Promoter DNA Methylation of miR-124 Promotes HIV-1 Tat-Mediated Microglial Activation via MECP2-STAT3 Axis. The Journal of neuroscience : the official journal of the Society for Neuroscience 2018, 38, 5367-5383, doi:10.1523/JNEUROSCI.3474-17.2018.

21. Hu, G.; Niu, F.; Liao, K.; Periyasamy, P.; Sil, S.; Liu, J.; Dravid, S.M.; Buch, S. HIV-1 Tat-Induced Astrocytic Extracellular Vesicle miR-7 Impairs Synaptic Architecture. Journal of neuroimmune pharmacology : the official journal of the Society on NeuroImmune Pharmacology 2020, 15, 538-553, doi:10.1007/s11481-019-09869-8.

22. Zhou, L.; Pupo, G.M.; Gupta, P.; Liu, B.; Tran, S.L.; Rahme, R.; Wang, B.; Rua, R.; Rizos, H.; Carroll, A.; et al. A parallel genome-wide mRNA and microRNA profiling of the frontal cortex of HIV patients with and without HIV-associated dementia shows the role of axon guidance and downstream pathways in HIV-mediated neurodegeneration. BMC genomics 2012, 13, 677, doi:10.1186/1471-2164-13-677.

23. Santerre, M.; Bagashev, A.; Gorecki, L.; Lysek, K.Z.; Wang, Y.; Shrestha, J.; Del Carpio-Cano, F.; Mukerjee, R.; Sawaya, B.E. HIV-1 Tat protein promotes neuronal dysregulation by inhibiting E2F transcription factor 3 (E2F3). The Journal of biological chemistry 2019, 294, 3618-3633, doi:10.1074/jbc.RA118.003744.

24. Ke, X.S.; Liu, C.M.; Liu, D.P.; Liang, C.C. MicroRNAs: key participants in gene regulatory networks. Current opinion in chemical biology 2003, 7, 516-523.

25. Bartel, D.P. MicroRNAs: genomics, biogenesis, mechanism, and function. Cell 2004, 116, 281-297, doi:10.1016/s00928674(04)00045-5.

26. Sempere, L.F.; Freemantle, S.; Pitha-Rowe, I.; Moss, E.; Dmitrovsky, E.; Ambros, V. Expression profiling of mammalian microRNAs uncovers a subset of brain-expressed microRNAs with possible roles in murine and human neuronal differentiation. Genome biology 2004, 5, R13, doi:10.1186/gb-2004-5-3-r13.

27. Podolska, A.; Kaczkowski, B.; Kamp Busk, P.; Sokilde, R.; Litman, T.; Fredholm, M.; Cirera, S. MicroRNA expression profiling of the porcine developing brain. PloS one 2011, 6, e14494, doi:10.1371/journal.pone.0014494.

28. Gao, F.B. Posttranscriptional control of neuronal development by microRNA networks. Trends Neurosci 2008, 31, 20-26, doi:10.1016/j.tins.2007.10.004. 
29. Fineberg, S.K.; Kosik, K.S.; Davidson, B.L. MicroRNAs potentiate neural development. Neuron 2009, 64, 303-309, doi:10.1016/j.neuron.2009.10.020.

30. McNeill, E.; Van Vactor, D. MicroRNAs shape the neuronal landscape. Neuron 2012, 75, 363-379, doi:10.1016/j.neuron.2012.07.005.

31. Rahimian, P.; He, J.J. HIV-1 Tat-shortened neurite outgrowth through regulation of microRNA-132 and its target gene expression. Journal of neuroinflammation 2016, 13, 247, doi:10.1186/s12974-016-0716-2.

32. Bagashev, A.; Mukerjee, R.; Santerre, M.; Del Carpio-Cano, F.E.; Shrestha, J.; Wang, Y.; He, J.J.; Sawaya, B.E. Involvement of miR-196a in HIV-associated neurocognitive disorders. Apoptosis : an international journal on programmed cell death 2014, 19, 1202-1214, doi:10.1007/s10495-014-1003-2.

33. Chaudhuri, A.D.; Yelamanchili, S.V.; Fox, H.S. MicroRNA-142 reduces monoamine oxidase A expression and activity in neuronal cells by downregulating SIRT1. PloS one 2013, 8, e79579, doi:10.1371/journal.pone.0079579.

34. Yelamanchili, S.V.; Chaudhuri, A.D.; Chen, L.N.; Xiong, H.; Fox, H.S. MicroRNA-21 dysregulates the expression of MEF2C in neurons in monkey and human SIV/HIV neurological disease. Cell death E disease 2010, 1, e77, doi:10.1038/cddis.2010.56.

35. Tatro, E.T.; Hefler, S.; Shumaker-Armstrong, S.; Soontornniyomkij, B.; Yang, M.; Yermanos, A.; Wren, N.; Moore, D.J.; Achim, C.L. Modulation of BK channel by MicroRNA-9 in neurons after exposure to HIV and methamphetamine. Journal of neuroimmune pharmacology : the official journal of the Society on NeuroImmune Pharmacology 2013, 8, 1210-1223, doi:10.1007/s11481-013-9446-8.

36. Xia, C.; Cai, Y.; Lin, Y.; Guan, R.; Xiao, G.; Yang, J. MiR-133b-5p regulates the expression of the heat shock protein 70 during rat neuronal cell apoptosis induced by the gp120 V3 loop peptide. Journal of medical virology 2016, 88, 437-447, doi:10.1002/jmv.24355.

37. Asahchop, E.L.; Branton, W.G.; Krishnan, A.; Chen, P.A.; Yang, D.; Kong, L.; Zochodne, D.W.; Brew, B.J.; Gill, M.J.; Power, C. HIV-associated sensory polyneuropathy and neuronal injury are associated with miRNA-455-3p induction. JCI insight 2018, 3, doi:10.1172/jci.insight.122450.

38. Eletto, D.; Russo, G.; Passiatore, G.; Del Valle, L.; Giordano, A.; Khalili, K.; Gualco, E.; Peruzzi, F. Inhibition of SNAP25 expression by HIV-1 Tat involves the activity of mir-128a. Journal of cellular physiology 2008, 216, 764-770, doi:10.1002/jcp.21452.

39. Chang, J.R.; Mukerjee, R.; Bagashev, A.; Del Valle, L.; Chabrashvili, T.; Hawkins, B.J.; He, J.J.; Sawaya, B.E. HIV-1 Tat protein promotes neuronal dysfunction through disruption of microRNAs. The Journal of biological chemistry 2011, 286, 41125-41134, doi:10.1074/jbc.M111.268466.

40. Mukerjee, R.; Chang, J.R.; Del Valle, L.; Bagashev, A.; Gayed, M.M.; Lyde, R.B.; Hawkins, B.J.; Brailoiu, E.; Cohen, E.; Power, C.; et al. Deregulation of microRNAs by HIV-1 Vpr protein leads to the development of neurocognitive disorders. The Journal of biological chemistry 2011, 286, 34976-34985, doi:10.1074/jbc.M111.241547.

41. Chou, C.H.; Shrestha, S.; Yang, C.D.; Chang, N.W.; Lin, Y.L.; Liao, K.W.; Huang, W.C.; Sun, T.H.; Tu, S.J.; Lee, W.H.; et al. miRTarBase update 2018: a resource for experimentally validated microRNA-target interactions. Nucleic acids research 2018, 46, D296-D302, doi:10.1093/nar/gkx1067.

42. Liu, W.; Wang, X. Prediction of functional microRNA targets by integrative modeling of microRNA binding and target expression data. Genome biology 2019, 20, 18, doi:10.1186/s13059-019-1629-z.

43. Agarwal, V.; Bell, G.W.; Nam, J.W.; Bartel, D.P. Predicting effective microRNA target sites in mammalian mRNAs. eLife 2015, 4, doi:10.7554/eLife.05005.

44. Shannon, P.; Markiel, A.; Ozier, O.; Baliga, N.S.; Wang, J.T.; Ramage, D.; Amin, N.; Schwikowski, B.; Ideker, T. Cytoscape: a software environment for integrated models of biomolecular interaction networks. Genome Res 2003, 13, 2498-2504, doi:10.1101/gr.1239303. 
45. Luo, W.; Brouwer, C. Pathview: an R/Bioconductor package for pathway-based data integration and visualization. Bioinformatics 2013, 29, 1830-1831, doi:10.1093/bioinformatics/btt285.

46. Hausser, J.; Zavolan, M. Identification and consequences of miRNA-target interactions--beyond repression of gene expression. Nat Rev Genet 2014, 15, 599-612, doi:10.1038/nrg3765.

47. Li, M.; Ransohoff, R.M. Multiple roles of chemokine CXCL12 in the central nervous system: a migration from immunology to neurobiology. Prog Neurobiol 2008, 84, 116-131, doi:10.1016/j.pneurobio.2007.11.003.

48. O'Meara, T.; Kong, Y.; Chiarella, J.; Price, R.W.; Chaudhury, R.; Liu, X.; Spudich, S.; Robertson, K.; Emu, B.; Lu, L. Exosomal MicroRNAs Associate With Neuropsychological Performance in Individuals With HIV Infection on Antiretroviral Therapy. J Acquir Immune Defic Syndr 2019, 82, 514-522, doi:10.1097/QAI.0000000000002187.

49. Fan, W.; Ye, G. Microarray analysis for the identification of specific proteins and functional modules involved in the process of hepatocellular carcinoma originating from cirrhotic liver. Mol Med Rep 2018, 17, 5619-5626, doi:10.3892/mmr.2018.8555.

50. Chin, C.H.; Chen, S.H.; Wu, H.H.; Ho, C.W.; Ko, M.T.; Lin, C.Y. cytoHubba: identifying hub objects and sub-networks from complex interactome. BMC systems biology 2014, 8 Suppl 4, S11, doi:10.1186/1752-0509-8-S4-S11.

51. Bindea, G.; Mlecnik, B.; Hackl, H.; Charoentong, P.; Tosolini, M.; Kirilovsky, A.; Fridman, W.H.; Pages, F.; Trajanoski, Z.; Galon, J. ClueGO: a Cytoscape plug-in to decipher functionally grouped gene ontology and pathway annotation networks. Bioinformatics 2009, 25, 1091-1093, doi:10.1093/bioinformatics/btp101.

52. Bindea, G.; Galon, J.; Mlecnik, B. CluePedia Cytoscape plugin: pathway insights using integrated experimental and in silico data. Bioinformatics 2013, 29, 661-663, doi:10.1093/bioinformatics/btt019.

53. Kanehisa, M.; Sato, Y.; Kawashima, M. KEGG mapping tools for uncovering hidden features in biological data. Protein Sci 2022, 31, 47-53, doi:10.1002/pro.4172. 